Received: 14 March 2017

Accepted: 28 June 2017

Published online: 04 August 2017
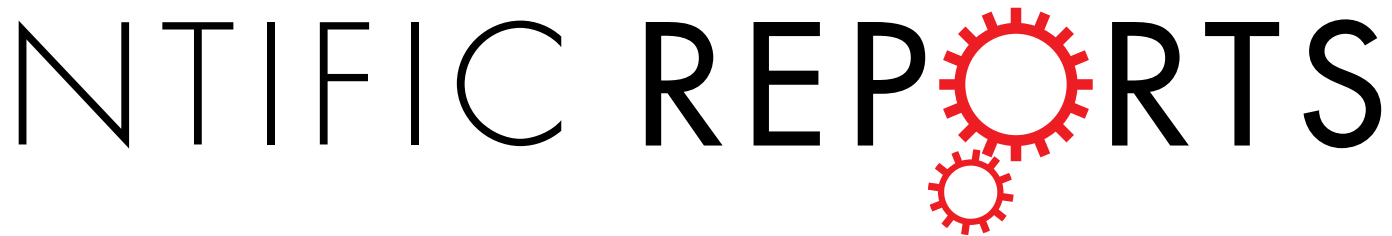

OPEN

\section{Aspergillus Secondary Metabolite Database, a resource to understand the Secondary metabolome of Aspergillus genus}

Varahalarao Vadlapudi ${ }^{1}$, Nabajyoti Borah ${ }^{1}$, Kanaka Raju Yellusani ${ }^{1}$, Sriramya Gade ${ }^{1}$, Prabhakar Reddy ${ }^{1}$, Maheshwari Rajamanikyam ${ }^{1}$, Lakshmi Narasimha Santosh Vempati ${ }^{1}$, Satya Prakash Gubbala ${ }^{1}$, Pankaj Chopra ${ }^{1,2}$, Suryanarayana Murty Upadhyayula ${ }^{2}$ \& Ramars Amanchy ${ }^{3}$

Aspergillus is a genus of ubiquitous fungi that are pathologically \& therapeutically important. Aspergillus Secondary Metabolites Database (A2MDB) is a curated compendium of information on Aspergillus \& its secondary metabolome. A2MDB catalogs 807 unique non-redundantsecondary metabolites derived from 675 Aspergillus species. A2MDB has a compilation of 100 cellular targets of secondary metabolites, 44 secondary metabolic pathways, 150 electron and light microscopy images of various Aspergillus species. A phylogenetic representation of over 2500 strains has been provided. A2MDB presents a detailed chemical information of secondary metabolites and their mycotoxins. Molecular docking models of metabolite-target protein interactions have been put together. A2MDB also has epidemiological data representing Aspergillosis and global occurrence of Aspergillus species. Furthermore a novel classification of Aspergillosis along with 370 case reports with images, were made available. For each metabolite catalogued, external links to related databases have been provided. All this data is available on A2MDB, launched through Indian Institute of Chemical Technology, Hyderabad, India, as an open resource http://www.iictindia.org/A2MDB. We believe A2MDB is of practical relevance to the scientific community that is in pursuit of novel therapeutics.

The initial description and nomenclature of Aspergillus was credited to Micheli (1729), Haller (1768) and Fries (1832) who proposed a generic name for a group of fungi sharing similar morphological characteristics ${ }^{1}$. Microscopic examination reveals a spore bearing structure conidiophore, having aspergillum-like morphology. Aspergillus species thrive as endophytes ${ }^{2}$, saprophytes ${ }^{3}$, parasites ${ }^{4}$ and also as human pathogens ${ }^{5-7}$. Aspergillus species cause localized and systemic human conditions termed as Aspergilloses ${ }^{8,9}$. They are also responsible for diseases in agricultural crops $^{10}$ and domestic animals ${ }^{10}$.

Fungi are categorized and classified based on morphology and molecular genetics. Morphological characterization of various Aspergillus spp. requires a catalog of microscopic images and genetic examination of clinical strains needs the sequence information of ITS regions. Aspergillus species although ubiquitous are more frequently observed in immuno-compromised individuals upon inhalation of conidia. Most usual complications are lung and cutaneous infections. Over the last decade there has been an increase in Aspergillosis reports, an emerging infectious disease that can be fatal. There is a need for better understanding of Aspergillosis and a resource containing a collection of case reports and a fresh classification is very much necessary, as information is scattered all over the literature.

${ }^{1}$ Pharmacology \& Toxicology Division, CSIR-Indian Institute of Chemical Technology (IICT), Uppal Road, Tarnaka, Hyderabad, 500 607, India. ${ }^{2}$ National Institute of Pharmaceutical Education \& Research (NIPER-Guwahati), 1st Floor, Institute of Pharmacy, Guwahati Medical College \& Hospital, Narakasur Hill top, Guwahati, 781 032, India. ${ }^{3}$ Division of Pharmacology \& Toxicology, CSIR-IICT (Indian Institute of Chemical Technology), Ministry of Science \& Technology (GOI), Uppal Road, Tarnaka, Hyderabad, Telangana, 500017, India. Varahalarao Vadlapudi, Nabajyoti Borah and Kanaka RajuYellusani contributed equally to this work. Correspondence and requests for materials should be addressed to S.M.U. (email: director@niperguwahati.ac.in) or R.A. (email: ramars@iict.res.in) 
Aspergillus spp. produces a wide range of structurally heterogeneous secondary metabolites. that are of considerable interest to the scientific research community. Fungi of this genus produce important secondary metabolites that have industrial importance ${ }^{11,12}$ and therapeutic significance like antibiotics ${ }^{13}$ and lovastatins ${ }^{14}$. As numerous natural products are being identified each day, a plethora of compounds still await discovery and a database can act as platform for their collection and annotation. Due to the need for Aspergillus centric metabolome repository, we have developed an open, user friendly resource; $A 2 \mathrm{MDB}$ that has experimental metabolomic data, catalogued and annotated with literature information. $A 2 \mathrm{MDB}$ provides an easy access to unbiased, comprehensive information about Aspergillosis, Aspergillus species, their secondary metabolites and cellular targets, molecular docking of metabolites-target interaction, secondary metabolic pathways, ITS based phylogeny and microscopic morphology. A2MDB also provides latest classification of Aspergillosis and collection of 370 case reports with over 70 reported variants of Aspergillosis. In the future more number of species, metabolite and molecular target data along with Aspergiloosis case reports will be included as and when additional information becomes available.

\section{A2MDB Database Development Data Mining.}

(a) Articles containing the search term Aspergillus (42189 articles as on 12/08/2016) and secondary metabolites (13770 articles as on 12/08/2016) were screened for cataloguing secondary metabolites from genus Aspergillus and this was collected with NCBI taxonomy ID and Mycobank ID.

(b) Microscopy images of Aspergillus species, Aspergillosis case reports and Secondary metabolite biosynthetic pathways were searched and collected from PubMed.

(c) ITS sequences of 7715 different Aspergillus species were collected from NCBI database for the primary analysis, out of which 2580 non-redundant sequences were from unique strains and species. The criterion for selection of a sequence was the availability of complete "18S-ITS1-5.8S-ITS2-28S" sequence. The non-redundant data was considered for the phylogenetic analysis.

(d) Multiple sequence alignment was performed using MAFFT $7^{15}$ and tree was constructed using UPGMA method. "iTOL" web interface was used for tree viewing and editing purpose ${ }^{16}$.

(e) All secondary metabolites archived in A2MDB have been linked to Public chemical compound databases ${ }^{17}$, PubChem $^{18}$, ChemSpider $^{19}$, TOXNET $^{20}$, ChEBI $^{21}$ and Chemical abstracts ${ }^{22}$. Classification of the 805 secondary metabolites has been done according to IUPAC nomenclature established and verified using NCBI-MeSH ${ }^{23}$.

(f) Tertiary structures of proteins described as cellular targets for the secondary metabolites were retrieved from Protein Data Bank (PDB). Protein Structures were optimized using Discovery Studio 4.5 Client ${ }^{24}$. The structures of secondary metabolites were downloaded from PubChem and optimized in Autodock ${ }^{25}$ and docked using AutoDock Vina ${ }^{26}$. The probable 3D models were generated using PyMOL ${ }^{27}$.

(g) A2MDB database technology: A2MDB database runs on MicroSoft.NET technology. ASP.NET and C\#.net technologies have been used to build the dynamic web interface. C\#, a server side scripting language, provides interface and assists in fetching data. ASP.NET Web pages function as HTML pages at run time. JavaScript was applied to ASP web pages for generating faster output with less stress on the server. A2MDB uses custom-designed lookup tables that ensure rapid responses to search queries. The relational architecture of $A 2 \mathrm{MDB}$ ensures data integrity and expandability, scope of the database. SQL server 2008 was used to facilitate back-end database support for storing the data and Asp.net as front end is used for fetching the data. Database has been provided a refined customized search functionality and search capability especially for the Aspergillus species, metabolites and docking images.

(h) Epidemiology: In order to visualize the global distribution of Aspergillus along with the Aspergillosis disease location, data mining was done from PubMed and based on the literature a global map was created using a R-package "rworldmap" (version $1.3-6)^{28}$ and spotting of the regions were done in Adobe Illustrator CC 2015, to showcase the incidence of Aspergillus spp. and Aspergillosis.

\section{Results and Discussion}

A2MDB is a database that is organized around the central entity, Aspergillus genus focusing primarily on its Secondary Metabolites and their biological interactions with the basic goal of understanding metabolic pathways in Aspergillus spp. and Aspergillosis (Fig. 1). A2MDB is a one of a kind resource that provides access to unique secondary metabolites produced by Aspergillus species. A2MDB is an efficient, non-redundant, user-friendly resource for viewing, sorting and extracting information. Each set of data is connected to every other set of data so that every possible aspect related to species, metabolite, metabolic pathways and cellular targets available so far, is brought together and can be downloaded.

A2MDB data retrieval, quality check and annotation. A2MDB is a curated secondary database to showcase metabolome of a group of fungi belonging to the genus Aspergillus. Information contained in it is, data related to Aspergillus ITS sequences, metabolites and their targets, electron microscopic images, information on Aspergillosis are based purely on text mining from PubMed and other authentic primary databases ${ }^{17,18,29}$.

Data entry in $A 2 \mathrm{MDB}$ follows a set protocol that enables (1) Identification of a new of species/strain with NCBI taxonomy ID, search and identification of any secondary metabolites with their chemical identifiers, (2) Collection of its genomic ITS data (3) search and identification of a metabolic target (4) metabolite-target interaction modeling (5) Search for a pathway (6) Search for microscopy information and further Aspergillosis 


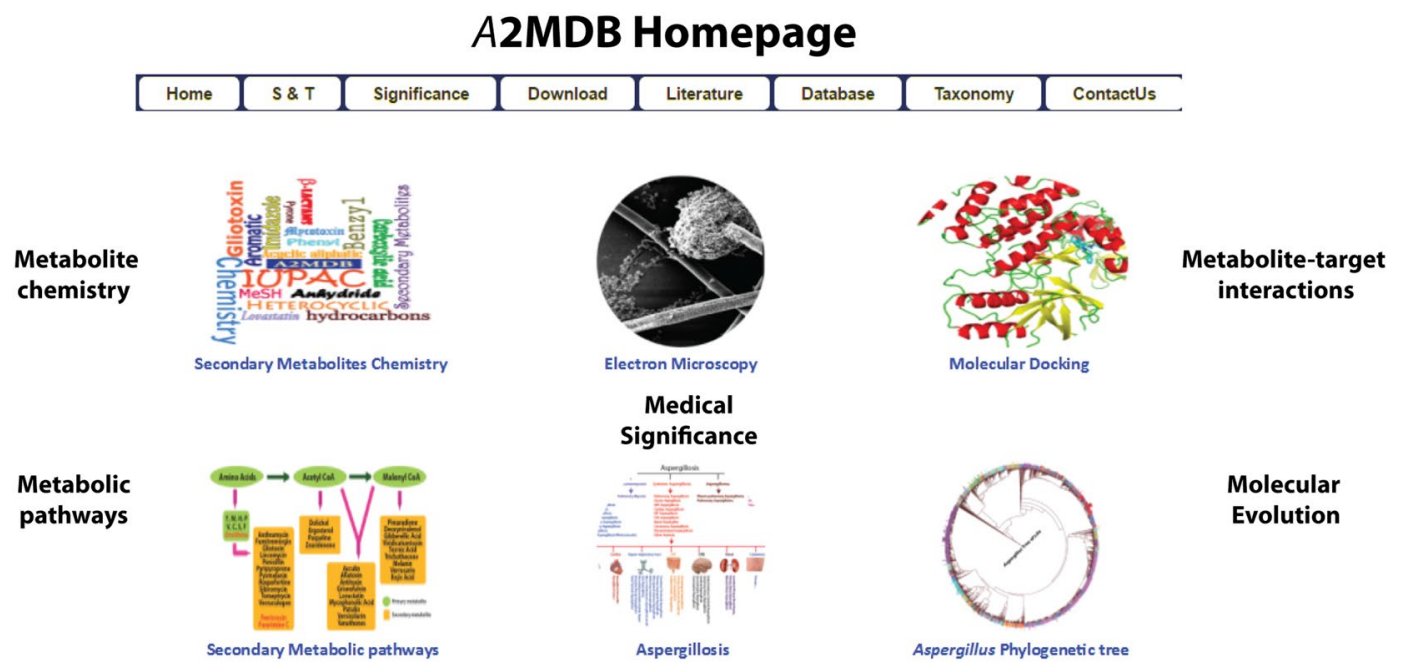

Figure 1. A screenshot of A2MDB showing multiple features in the homepage. The figure shows how clicking on various icons can navigate to the required option. Main page has icons for secondary metabolites chemistry, biological targets, Secondary metabolic pathways, microscopy images, molecular docking, Aspergillosis and phylogenetic analysis, apart from buttons which when clicked, explain science and significance of the project, database pages where one can surf and download data, taxonomy and contact us pages. The database has been officially launched through CSIR-IICT, India webpages.

components. In the Data curation of $A 2 \mathrm{MDB}$, all the data has been verified by a curator besides the contributor and an additional scientist.

The quality of the data coming from a publication has already been peer reviewed and hence without bias full reference of the publication has been given for each and every piece of gathered data that has been provided. For quality check during curation of the data, Iwe generally follow a three step strategy.

(1) Data collection: - The text mining is done only in the authenticated primary database and those data having valid reference are collected for entry in the A2MDB after being verified by 2 qualified scientists.

(2) Pre-entry review: - In this step we follow the simple duplicate data entry validation system. For this purpose two independent files are produced for each dataset and these are compared to check for any discrepancy by two different scientists. After removing discrepancy and redundancy a final dataset is generated for entry to the database.

(3) Post-entry review: - After the final tables were prepared, we check for any missing data and then a random sample of data is audited against the entered data to find manual errors.

The newer data coming in either by our group or externally will be entered after the three step cycle.

The data retrieval from primary databases although performed manually, was carried out with a systematic approach that dramatically reduced the work load in manual reviewing. We used the boolean and field operators in PubMed search ${ }^{30}$ to make our raw dataset as accurate as possible. Screening or pre-processing of the literature dataset was based on standard text mining procedure and after the data was collected it was annotated by scientists and uploaded and verified before making the database public.

Searching and browsing A2MDB. A2MDB web application allows users to search or browse for Aspergillus Species resources by either specifying a search string or by choosing different optional filters to species name, metabolites, biological function, cellular target, PMID and Tax ID links. Search results are usually displayed as a tabular format and some browsable elements as PDF format. All the data available through A2MDB is downloadable and the content is available as tables as well as PDF files. A2MDB application also allows users to view all results on the same page at one time. The table consists of columns showing, for each resource, its species name, metabolites, biological function, cellular target, PMID and NCBI Taxonomy ID. In addition to providing comprehensive data, each metabolite and cellular target also contains hyperlinks to other authentic databases (PubChem, PubMed), references, digital images and applets for viewing directly from the primary database. Docking images are shown as image objects that show details of docking upon clicking the object.

Metabolite information and classification. A2MDB has a collection of about 675 Aspergillus species, linked to a Taxonomy ID in NCBI database. 581 species have been annotated with links to Mycobank. 807 unique metabolites isolated from 324 species of this genus are incorporated so far with the objective of providing complete chemical and biological target related information. Nearly 25 species were identified for their variety of secondary metabolies produced. In an attempt to give complete chemical information, 523 of these secondary metabolites were connected to chemical databases like PubChem, ChemSpider, ChEBI, TOXNET. Among the secondary metabolites 213 were isolates from A. flavus, A. fumigatus, A. oryzae and A. niger of which 90 were 

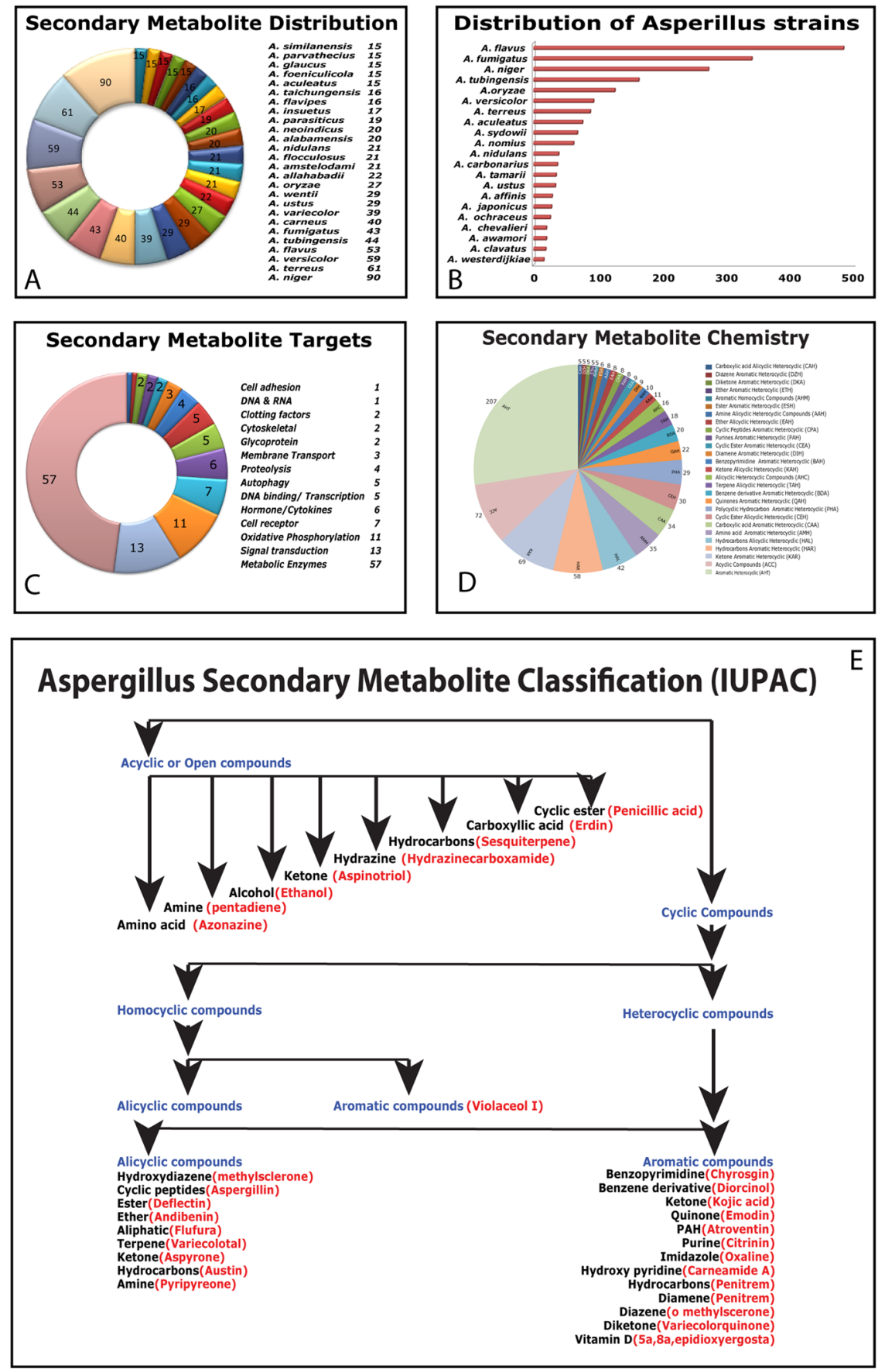

Figure 2. Analysis of the data collected for assimilation in to A2MDB: (A) pie chart representing the number of metabolites identified from major Aspergillus species, (B) bar graph shows the clinical distribution of biologically, medically and important strains of major Aspergillus species, (C) gene ontology based classification of cellular targets based on molecular function, (D) Distribution of secondary metabolites based on their chemical property, (E) IUPAC based classification of secondary metabolites in to various chemical compounds shown with examples under each category.

from A. niger alone (Fig. 2A). This culmination among the highly enriched ones, is because these species are most studied owing to their etiological importance of some of the species.

Out of the 807 metabolites identified, majority of the secondary metabolites have been identified from around 25 Aspergillus species (Fig. 2A). 60 different Mycotoxins were identified (Supplementary Table 1) from 35 species of Aspergillus that pose a considerable amount of threat to veterinary and human health ${ }^{31}$. Aflatoxins produced by Aspergillus flavus and Aspergillus parasiticus have been known to be carcinogenic and hepatotoxic in nature. Aflatoxin $\mathrm{B} 1$ the most toxic mycotoxin can penetrate through the skin $^{32}$. Ochratoxin, contaminant of cereals, is primarily produced by Aspergillus ochraceus ${ }^{33}$ causes liver damage, enterititis, immunosuppression, 

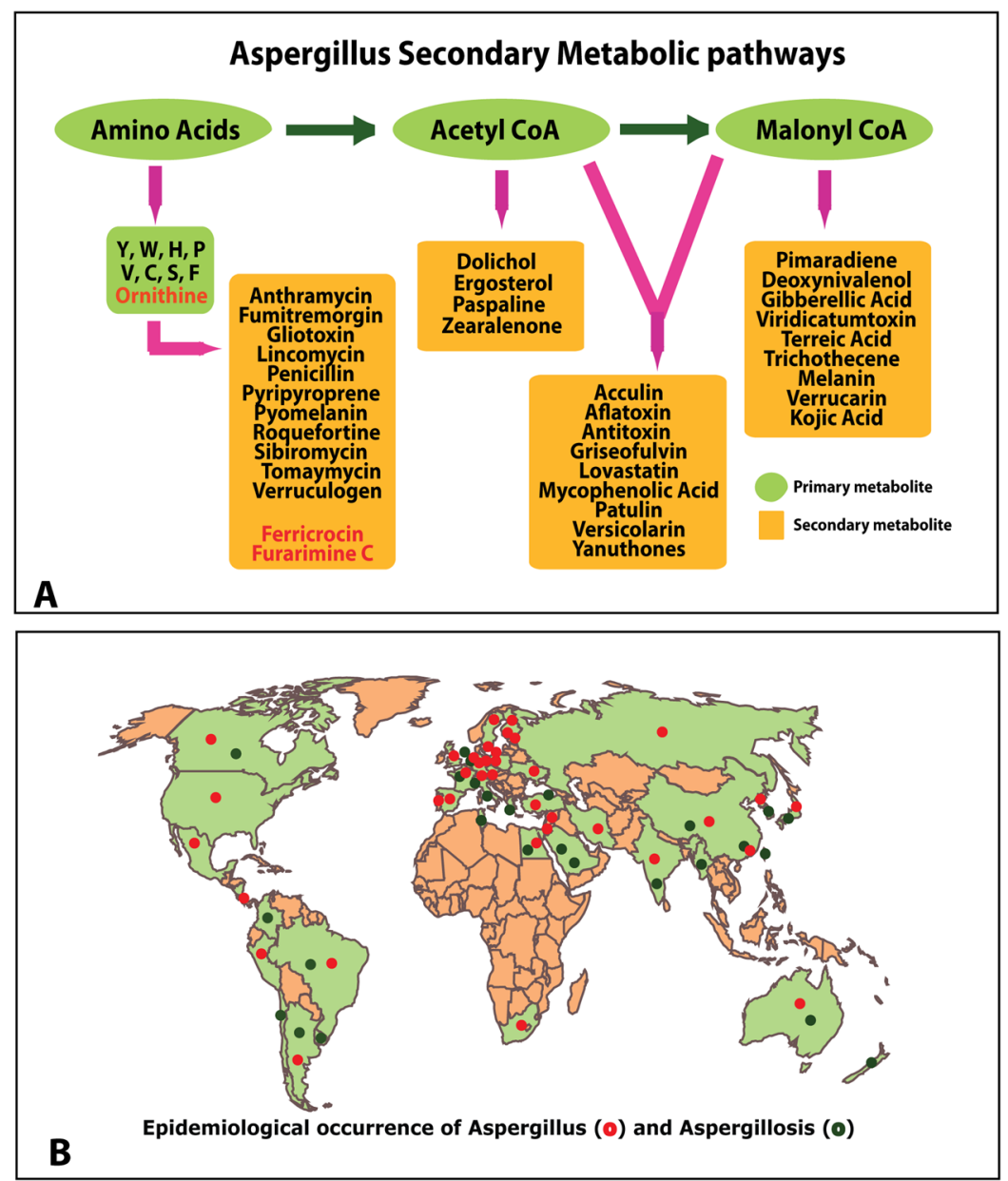

Figure 3. (A) A representation of metabolic pathways where in the primary metabolic intermediates serve as precursors for secondary metabolite production in Aspergillus, (B) A qualitative epidemiological distribution map was created using a R-package "rworldmap" (version 1.3-6) to showcase the incidence of Aspergillus spp. and Aspergillosis.

teratogenesis, nephrotoxicity and renal tumors ${ }^{31}$ Citrinin is a polyketide mycotoxin, produced by Aspergillus candidus and Aspergillus carneus shows nephrotoxic, hepatotoxic and cyototoxic behaviour. Another lethal mycotoxin sterigmatocystin is mainly found in dairy products, is a possible carcinogen mainly produced by Aspergillus nidulans and Aspergillus versicolor. Patulin is a mycotoxin produced by Aspergillus giganteus usually associated with spoilage of apple and grapes. causes cerebral hemorrhage. Within each species, several biologically important strains; were reported that are either pathogenic, or that are industrially or biologically important (Fig. 2B). A vast majority of strains that are clinically, biolocically and industrially important were identified to be from 18 species (Fig. 2B) which not only shows the infectious nature of these species in producing toxins but also of their industrial importance by the nature of the important secondary metabolites produced. We observed that majority of these secondary metabolites had been found to be targeting metabolic enzymes in order to manipulate cellular machinery (Fig. 2C). These metabolites have been linked to a unique registry number provided by Chemical Abstracts Service (CAS) of the American Chemical Society ${ }^{22}$ and classified into categories based on IUPAC nomenclature (Fig. 2D,E). Whole genome sequencing of Aspergillus species has pointed out to a varying diversity of the enzymes involved in the secondary metabolism and a range of novel compounds remain elusive and uncharacterized ${ }^{34}$. Analysis is ongoing to identify secondary metabolism gene clusters in over 250 species of Aspergillus.

Secondary metabolic pathways. Primary metabolism is well studied in fungi and well documented in many databases and secondary metabolites and metabolic pathways in fungi are underrepresented in databases. Precursors derived from the primary metabolic pathways are siphoned into secondary metabolic pathways to synthesize compounds that have unusual structures and biological properties (Figs 2D and 3A). There is an increasing need to understand secondary metabolism to exploit these organisms, and control the production of potential drugs and toxins. In $A 2 \mathrm{MDB}$ we provide 44 secondary metabolic pathway illustrations directly taken from the literature with references. 133 biological targets for 135 of these metabolites have been identified from the literature so far. We have also furnished external links to 1578 metabolic pathways from KEGG database that are Aspergillus specific (1447 primary and 131 secondary metabolic pathways). We have also provided 


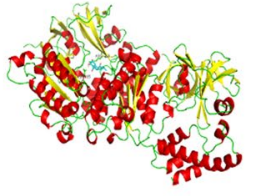

Dehydrocurvularin - P97

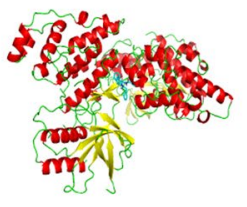

Asperphenamate - JNK

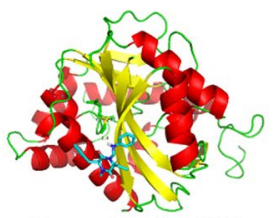

Ditryptophenaline - NMT

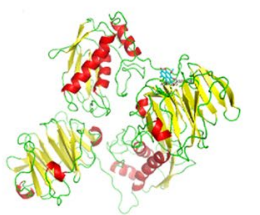

Funalenone-Colagenase I

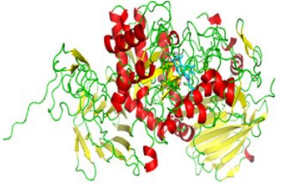

Aspergillin PZ - Glusosidase

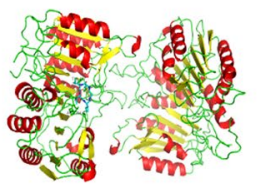

MalforminA1 - Caspase 3

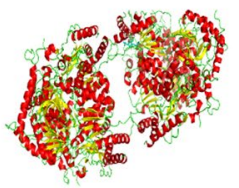

Flavoglaucin - LDH

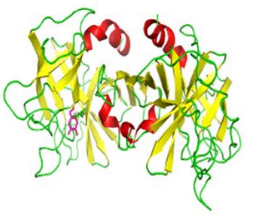

kojic acid-Neutrophil elastase
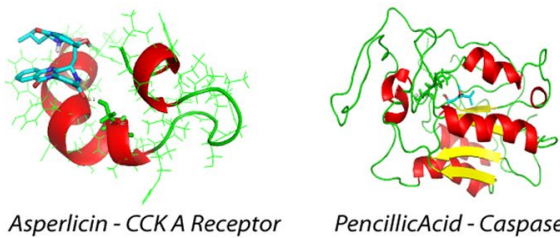

PencillicAcid - Caspase 8

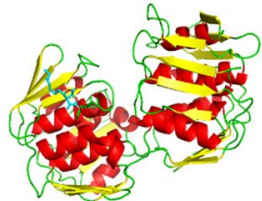

Avenaciolide - Mur A

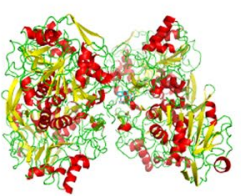

Flavasperone-Cholesterol acyl transferase

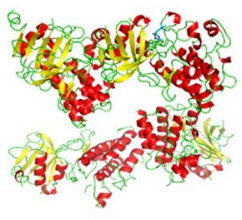

NeoechinulinA - NFkB

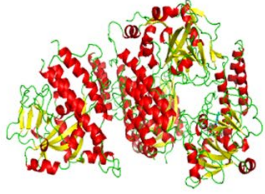

Quinazolinone-PARP

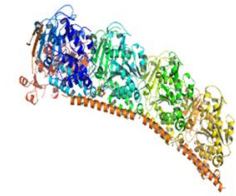

Neoxiline - tubulin

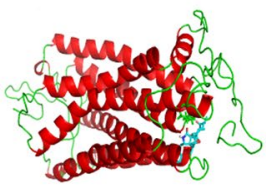

Rosellichalasin - NK1 receptor

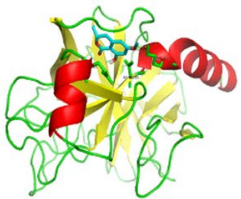

6methoxymellein - Kallikrein5

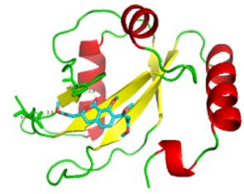

Viriditoxin - LC3

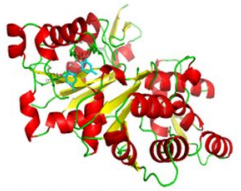

Violaceiol1 - G-actin

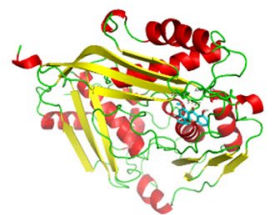

Sterigmatocystin - SAM synthetase

\section{Secondary metabolite - cellular target interactions}

Figure 4. Molecular docking models representing secondary metabolite and cellular target interactions as reported in literature. Docking was performed using Auto Dock Vena. The protein structure was from PDB, metabolite structure from one of the chemical databases as explained in the text and binding energies are also represented.

information about anti-fungal compounds and their bioactivity on Aspergillus species, with relevant information from $\mathrm{ChEMBL}^{35}$.

Epidemiology. As Aspergillus produces small hydrophobic conidia that easily disperse into air and can survive in the drastic environmental condition, distribution of Aspergillus species is found to be ubiquitous across different ecological niche ${ }^{3}$. The geographical map was provided that provides clear correlation of the occurrence of the pathogen and disease (Fig. 3B).

Molecular modelling of secondary metabolite - target interactions were provided to further validate their likely metabolite-target interactions with PDB structures based on docking energies and binding affinities (Fig. 4). Further studies are being carried out to understand their structure activity relationships.

Molecular and Microscopic examination. In A2MDB we provide 223 Microscopy images of Aspergillus spp.; a collection of literature derived electron and light microscopy images is provided with links to original published articles. Morphological information by means of optical and electron microscopy images as well as molecular phylogenetic information by means of ITS sequences as reported earlier was provided.

Aspergillosis classification and Case studies. Aspergillosis caused by various Aspergillus spp. has been classified broadly in to 5 different categories and over 70 different types (Fig. 5 ) that fall under 5 primary categories (invasive aspergillosis, pneumomycosis, systemic aspergillosis, aspergilloma and allergic aspergillosis. Aspergillosis in humans and animals has been mentioned in depth using case reports and CT scan images wherever available from 370 PubMed articles each one available for download with article identifier.

Taxonomical information. A phylogenetic tree of life based on 2580 ITS sequences, selected from 175 unique strains and species of Aspergillus (Fig. 6) was represented. A significant number of Aspergillus species can be associated with 11 different sexual stages (teleomorphs); Emericella, Eurotium, Fennellia, Neosartorya, Petromyces, Dichotomomyces, Chaetosartorya, Cristaspora, Penicilliopsis, Sclerocleista and Phialosimplex that have now come under the umbrella of Aspergillus after the decision of "one name one fungi" by International Code of Nomenclature. U.S. Department of Energy, Joint Genome Institute (DOE-JGI) has about 270 Aspergillus species/ 


\section{Classification of Aspergillosis}

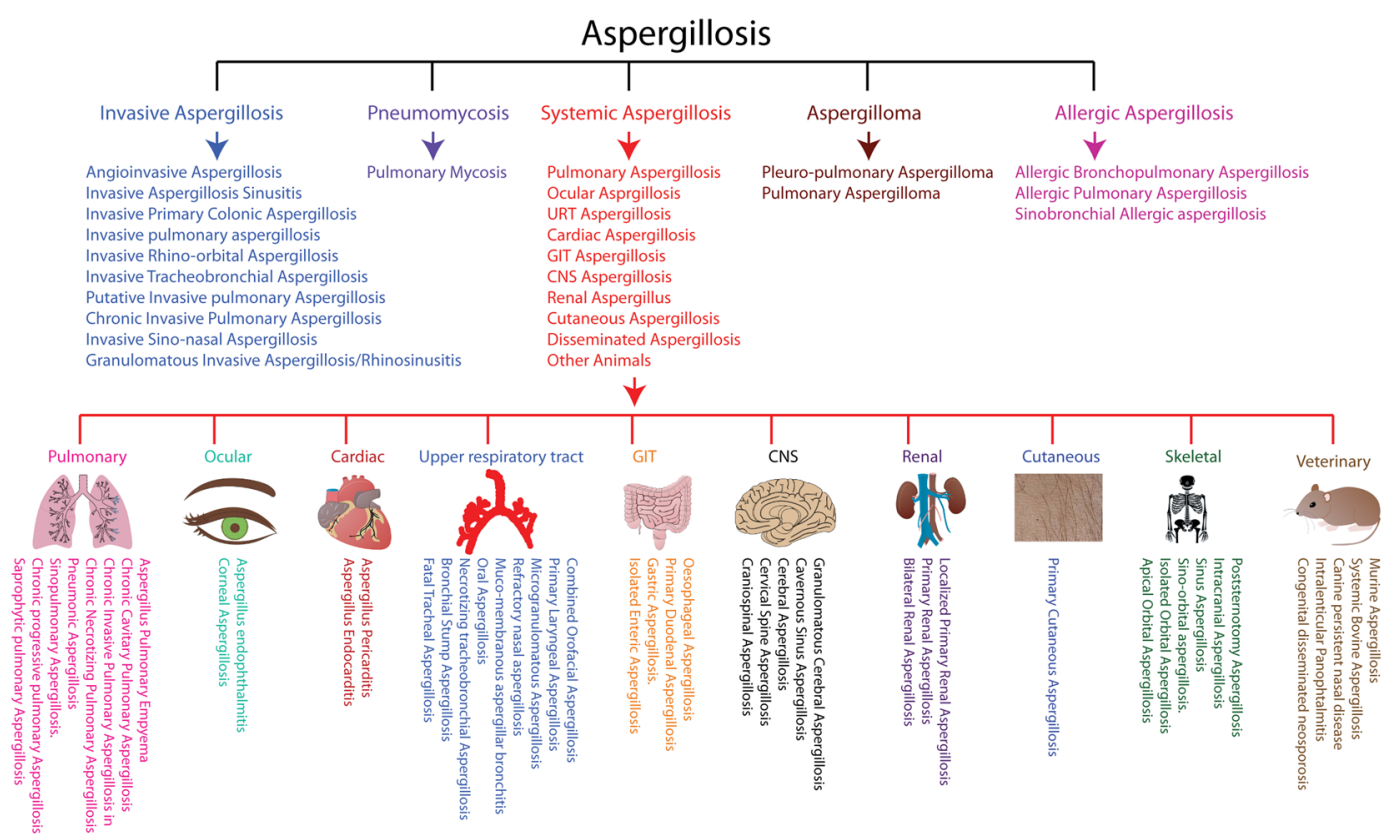

Figure 5. A detailed classification of Aspergillosis with case reports and related images from the literature available so far from humans and animals with DOI literature references. All the organ systems have been drawn using the software Edraw (Version 8.6) trial version.
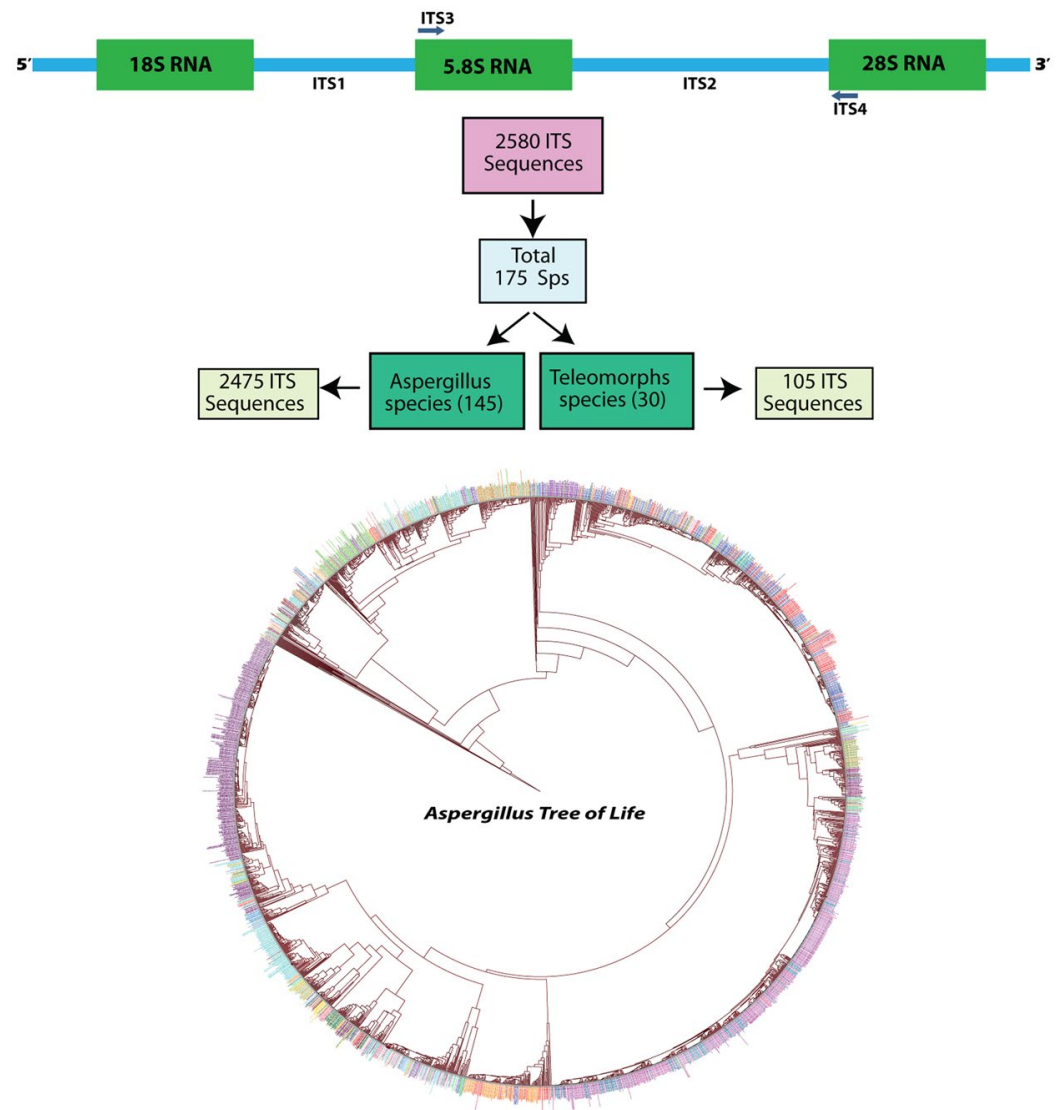

Figure 6. Phylogenetic tree of life for 2850 Aspergillus strains using ITS sequences. All the sequences are unique, various strains of Aspergillus belong mainly to 175 species. 
strains/teleomorphs in their genome sequencing list, out of which nearly 90 have been sequenced and the others in either sequencing pipeline or in review as part of 1000 fungal genome project.

Comparison with other fungal and metabolic databases. A2MDB is a non-redundant comprehensive compilation of Aspergillus specific secondary metabolism information. There are few databases that provide species specific information and even fewer on secondary metabolism and secondary metabolites of biological importance. While databases like PHI-base ${ }^{36}$, EHFPI ${ }^{37}$, catalogue genetic information to aide pathogenicity studies, ITS2 ${ }^{38}$, metaMicrobesOnline ${ }^{39}$, Mycobank ${ }^{29}$ provide for phylogenetic and taxonomic information. Primary metabolic pathways in eukaryotes are well annotated by databases like $\mathrm{KEGG}^{40}$, Reactome ${ }^{41}$ and $\mathrm{HMDB}^{42}$, while Metacyc, Biocyc and Humancyc are pathway genome databases ${ }^{43,44}$. Metacyc provides a list of natural compounds but is not organism specific. AntiSMASH ${ }^{45}$ and SMURF ${ }^{46}$ are web applications that predict bacterial and fungal secondary metabolic gene clusters ${ }^{45}$. Databases like YEASTRACT ${ }^{47}$, PomBase ${ }^{48}$, NetwoRx ${ }^{49}$ are specific to Saccharomyces species, but not to secondary metabolite/metabolism information. 'Aspergillus and Aspergillosis' resource provides secondary metabolite information from Aspergillus species, but redundancy exists and sources remain undefined with major focus on aspergillosis ${ }^{50}$ and is currently merged with Central Aspergillus Data REpository (CADRE) ${ }^{51}$. CADRE is a repository of genomic data of Aspergillus species ${ }^{51}$. Aspergillus Genome Database (AspGD) is a genetic information resource specific to Aspergillus spp. ${ }^{52}$. However, $A 2 M D B$ is a unique database that provides comprehensive secondary metabolite information related to vast number of Aspergillus species with resourceful evidence based information pertaining to Aspergillosis, Aspergillus specific anti-fungal compounds, ATCC Aspergillus collection, secondary metabolic pathways, phylogeny and other related databases. We believe that $A 2 M D B$ will find a global niche with its unique content.

\section{Future Directions.}

1. A2MDB has documented 807 secondary metabolites and almost 500 of these have chemical information from established chemical databases and we are trying to provide hyperlinks for the rest of the compounds to well established chemical and metabolite databases. Search for new metabolites is being actively done from the 351 species, that do not have prior reports on secondary metabolite isolation.

2. A2MDB has 370 case reports with images from 70 different types of Aspergillosis that was classified afresh. We are also trying to find information about Aspergillus causing diseases in plants.

3. Since some of the species are endophytes we are trying to identify and categorize Aspergillus species as endophytes and relationship with their host plants.

4. More species are being added along with their electron and light microscopy images as and when they become available.

5. Addition of transformation products of Aspergillus and derivatives of its secondary metabolites is under progress as well as their pathway annotation.

6. Molecular docking studies are being attempted using QSAR that might result in discovery of new therapeutic compounds.

7. Analysis is ongoing on identification of secondary metabolism gene clusters in all of the genome sequenced Aspergillus species.

8. Identification of primary metabolite that becomes the parent compound for the secondary metabolite is also being carried out.

\section{Conclusions}

$A 2 \mathrm{MDB}$ is a unique non-redundant resource of secondary metabolites and pathways information dedicated to Aspergillus species and Aspergillosis. A2MDB is regularly updated by database administrators and scientists. We welcome community participation in depositing and sharing the data. A2MDB is available for free without any registration. We believe $A 2 \mathrm{MDB}$ will be of immense importance to mycologists as well as scientists looking for important natural products obtained from Aspergillus species.

Data Accessibility. All the data being reported in this manuscript that has been collected, curated and deposited has been made publicly available directly through our database in the database download options (http://www.iictindia.org/a2mdb).

\section{References}

1. Samson, R. A. et al. Phylogeny, identification and nomenclature of the genus Aspergillus. Studies in mycology 78, 141-173, doi:10.1016/j.simyco.2014.07.004 (2014).

2. You, Y. H., Kwak, T. W., Kang, S. M., Lee, M. C. \& Kim, J. G. Aspergillus clavatus Y2H0002 as a New Endophytic Fungal Strain Producing Gibberellins Isolated from Nymphoides pe ltata in Fresh Water. Mycobiology 43, 87-91, doi:10.5941/MYCO.2015.43.1.87 (2015).

3. Dagenais, T. R. \& Keller, N. P. Pathogenesis of Aspergillus fumigatus in Invasive Aspergillosis. Clinical microbiology reviews 22, 447-465, doi:10.1128/CMR.00055-08 (2009).

4. Ogawa, A. et al. Pseudodeflectusin, a novel isochroman derivative from Aspergillus pseudodeflectus a parasite of the sea weed, Sargassum fusiform, as a selective human cancer cytotoxin. Bioorganic \& medicinal chemistry letters 14, 3539-3543, doi:10.1016/j. bmcl.2004.04.050 (2004).

5. Hedayati, M. T., Pasqualotto, A. C., Warn, P. A., Bowyer, P. \& Denning, D. W. Aspergillus flavus: human pathogen, allergen and mycotoxin producer. Microbiology 153, 1677-1692, doi:10.1099/mic.0.2007/007641-0 (2007).

6. Behnsen, J. et al. The opportunistic human pathogenic fungus Aspergillus fumigatus evades the host complement system. Infection and immunity 76, 820-827, doi:10.1128/IAI.01037-07 (2008). 
7. Paulussen, C. et al. Ecology of aspergillosis: insights into the pathogenic potency of Aspergillus fumigatus and some other Aspergillus species. Microbial biotechnology, doi:10.1111/1751-7915.12367 (2016).

8. Sivak-Callcott, J. A. et al. Localised invasive sino-orbital aspergillosis: characteristic features. The British journal of ophthalmology 88, 681-687 (2004)

9. Aleksenko, A. \& Gyasi, R. Disseminated invasive aspergillosis. Ghana medical journal 40, 69-72 (2006).

10. Perrone, G. et al. Biodiversity of Aspergillus species in some important agricultural products. Studies in mycology 59, 53-66, doi:10.3114/sim.2007.59.07 (2007).

11. Yoon, J., Kikuma, T., Maruyama, J. \& Kitamoto, K. Enhanced production of bovine chymosin by autophagy deficiency in the filamentous fungus Aspergillus oryzae. PloS one 8, e62512, doi:10.1371/journal.pone.0062512 (2013).

12. Singh, B. K. et al. Kojic Acid Peptide: A New Compound with Anti-Tyrosinase Potential. Annals of dermatology 28, 555-561, doi:10.5021/ad.2016.28.5.555 (2016).

13. Then Bergh, K. \& Brakhage, A. A. Regulation of the Aspergillus nidulans penicillin biosynthesis gene acvA (pcbAB) by amino acids: implication for involvement of transcription factor PACC. Applied and environmental microbiology 64, 843-849 (1998).

14. Fu, Y., Wu, P., Xue, J., Wei, X. \& Li, H. Versicorin, a new lovastatin analogue from the fungus Aspergillus versicolor SC0156. Natural product research 29, 1363-1368, doi:10.1080/14786419.2015.1026342 (2015).

15. Katoh, K. \& Standley, D. M. MAFFT multiple sequence alignment software version 7: improvements in performance and usability. Molecular biology and evolution 30, 772-780, doi:10.1093/molbev/mst010 (2013).

16. Letunic, I. \& Bork, P. Interactive Tree Of Life v2: online annotation and display of phylogenetic trees made easy. Nucleic acids research 39, W475-478, doi:10.1093/nar/gkr201 (2011).

17. Williams, A. J. Public chemical compound databases. Current opinion in drug discovery \& development 11, 393-404 (2008).

18. Kim, S. et al. PubChem Substance and Compound databases. Nucleic acids research 44, D1202-1213, doi:10.1093/nar/gkv951 (2016).

19. Editorial: ChemSpider-a tool for Natural Products research. Natural product reports 32, 1163-1164, doi:10.1039/c5np90022k (2015).

20. Wexler, P. TOXNET: an evolving web resource for toxicology and environmental health information. Toxicology 157, 3-10 (2001).

21. Hastings, J. et al. ChEBI in 2016: Improved services and an expanding collection of metabolites. Nucleic acids research 44, D1214-1219, doi:10.1093/nar/gkv1031 (2016).

22. Buntrock, R. E. Chemical registries-in the fourth decade of service. Journal of chemical information and computer sciences 41, 259-263 (2001)

23. Sartor, M. A. et al. Metab2MeSH: annotating compounds with medical subject headings. Bioinformatics 28, 1408-1410, doi:10.1093/ bioinformatics/bts156 (2012).

24. Brooks, B. R. et al. CHARMM: the biomolecular simulation program. Journal of computational chemistry 30, 1545-1614, doi:10.1002/jcc.21287 (2009).

25. Forli, S. et al. Computational protein-ligand docking and virtual drug screening with the AutoDock suite. Nature protocols 11 , 905-919, doi:10.1038/nprot.2016.051 (2016).

26. Trott, O. \& Olson, A. J. AutoDock Vina: improving the speed and accuracy of docking with a new scoring function, efficient optimization, and multithreading. Journal of computational chemistry 31, 455-461, doi:10.1002/jcc.21334 (2010).

27. DeLano, W. L. Unraveling hot spots in binding interfaces: progress and challenges. Current opinion in structural biology 12, 14-20 (2002).

28. R-Core-Team. R: A language and environment for statistical computing. R Foundation for Statistical Computing, Vienna, Austria. https://www.R-project.org/ (2016).

29. Robert, V. et al. MycoBank gearing up for new horizons. IMA fungus 4, 371-379, doi:10.5598/imafungus.2013.04.02.16 (2013).

30. Ebbert, J. O., Dupras, D. M. \& Erwin, P. J. Searching the medical literature using PubMed: a tutorial. Mayo Clinic proceedings 78, 87-91, doi:10.4065/78.1.87 (2003).

31. Brase, S., Encinas, A., Keck, J. \& Nising, C. F. Chemistry and biology of mycotoxins and related fungal metabolites. Chemical reviews 109, 3903-3990, doi:10.1021/cr050001f (2009).

32. Patten, R. C. Aflatoxins and disease. The American journal of tropical medicine and hygiene 30, 422-425 (1981).

33. Kolakowski, B., O’Rourke, S. M., Bietlot, H. P., Kurz, K. \& Aweryn, B. Ochratoxin A Concentrations in a Variety of Grain-Based and Non-Grain-Based Foods on the Canadian Retail Market from 2009 to 2014. Journal of food protection 79, 2143-2159, doi:10.4315/0362-028X.JFP-16-051 (2016).

34. Craney, A., Ahmed, S. \& Nodwell, J. Towards a new science of secondary metabolism. The Journal of antibiotics 66, 387-400, doi:10.1038/ja.2013.25 (2013)

35. Gaulton, A. et al. The ChEMBL database in 2017. Nucleic acids research, doi:10.1093/nar/gkw1074 (2016).

36. Urban, M. et al. The Pathogen-Host Interactions database (PHI-base): additions and future developments. Nucleic acids research 43, D645-655, doi:10.1093/nar/gku1165 (2015)

37. Liu, Y. et al. EHFPI: a database and analysis resource of essential host factors for pathogenic infection. Nucleic acids research $\mathbf{4 3}$, D946-955, doi:10.1093/nar/gku1086 (2015)

38. Koetschan, C. et al. The ITS2 Database III-sequences and structures for phylogeny. Nucleic acids research 38, D275-279, doi:10.1093/ nar/gkp966 (2010).

39. Chivian, D., Dehal, P. S., Keller, K. \& Arkin, A. P. MetaMicrobesOnline: phylogenomic analysis of microbial communities. Nucleic acids research 41, D648-654, doi:10.1093/nar/gks1202 (2013).

40. Kanehisa, M. et al. Data, information, knowledge and principle: back to metabolism in KEGG. Nucleic acids research 42, D199-205, doi:10.1093/nar/gkt1076 (2014).

41. Fabregat, A. et al. The Reactome pathway Knowledgebase. Nucleic acids research 44, D481-487, doi:10.1093/nar/gkv1351 (2016).

42. Wishart, D. S. et al. HMDB 3.0-The Human Metabolome Database in 2013. Nucleic acids research 41, D801-807, doi:10.1093/nar/ gks1065 (2013)

43. Caspi, R. et al. The MetaCyc database of metabolic pathways and enzymes and the BioCyc collection of pathway/genome databases. Nucleic acids research 44, D471-480, doi:10.1093/nar/gkv1164 (2016).

44. Romero, P. et al. Computational prediction of human metabolic pathways from the complete human genome. Genome biology 6, R2, doi:10.1186/gb-2004-6-1-r2 (2005).

45. Medema, M. H. et al. antiSMASH: rapid identification, annotation and analysis of secondary metabolite biosynthesis gene clusters in bacterial and fungal genome sequences. Nucleic acids research 39, W339-346, doi:10.1093/nar/gkr466 (2011).

46. Khaldi, N. et al. SMURF: Genomic mapping of fungal secondary metabolite clusters. Fungal genetics and biology: FG \& B 47, 736-741, doi:10.1016/j.fgb.2010.06.003 (2010)

47. Teixeira, M. C. et al. The YEASTRACT database: an upgraded information system for the analysis of gene and genomic transcription regulation in Saccharomyces cerevisiae. Nucleic acids research 42, D161-166, doi:10.1093/nar/gkt1015 (2014).

48. McDowall, M. D. et al. PomBase 2015: updates to the fission yeast database. Nucleic acids research 43, D656-661, doi:10.1093/nar/ gku1040 (2015).

49. Fortney, K. et al. NetwoRx: connecting drugs to networks and phenotypes in Saccharomyces cerevisiae. Nucleic acids research 41, D720-727, doi:10.1093/nar/gks1106 (2013). 
50. Mabey Gilsenan, J. E. et al. Aspergillus genomes and the Aspergillus cloud. Nucleic acids research 37, D509-514, doi:10.1093/nar/ gkn876 (2009).

51. Mabey Gilsenan, J., Cooley, J. \& Bowyer, P. CADRE: the Central Aspergillus Data REpository 2012. Nucleic acids research 40, D660-666, doi:10.1093/nar/gkr971 (2012).

52. Cerqueira, G. C. et al. The Aspergillus Genome Database: multispecies curation and incorporation of RNA-Seq data to improve structural gene annotations. Nucleic acids research 42, D705-710, doi:10.1093/nar/gkt1029 (2014).

\section{Acknowledgements}

VVR would like to thank to Ministry of Earth Sciences, New Delhi, India (MoES/36/OOIS/Extra/18/2013) for financial support towards Research Associateship. MR would like to thank Department of Science and Technology, India for the DST Inspire fellowship (GAP-0347). SG thanks Council of Scientific \& Industrial Research (CSIR), India for the support received in the form of Ph. D studentship (P811101). We thank Sri A. Radhakrishna, CSIR-IICT computer centre for technical help regarding SQL Server database management. We also thank the Director, IICT for granting us permission to launch A2MDB database through CSIR-IICT webpages.

\section{Author Contributions}

Research design: R.A., V.V., N.B.; Data mining: V.V., N.B., M.R., S.G., P.C.; Data analysis and annotation: N.B., R.A.; Molecular Docking: K.R.Y., V.V.; Phylogeny: N.B.; Database development: K.R.Y., P.R., S.P.G.; Contribution of tools: V.L.N.S., U.S.N.M.; Manuscript preparation: R.A., V.V., N.B.

\section{Additional Information}

Supplementary information accompanies this paper at doi:10.1038/s41598-017-07436-W

Competing Interests: The authors declare that they have no competing interests.

Publisher's note: Springer Nature remains neutral with regard to jurisdictional claims in published maps and institutional affiliations.

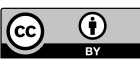

Open Access This article is licensed under a Creative Commons Attribution 4.0 International License, which permits use, sharing, adaptation, distribution and reproduction in any medium or format, as long as you give appropriate credit to the original author(s) and the source, provide a link to the Creative Commons license, and indicate if changes were made. The images or other third party material in this article are included in the article's Creative Commons license, unless indicated otherwise in a credit line to the material. If material is not included in the article's Creative Commons license and your intended use is not permitted by statutory regulation or exceeds the permitted use, you will need to obtain permission directly from the copyright holder. To view a copy of this license, visit http://creativecommons.org/licenses/by/4.0/.

(c) The Author(s) 2017 